\title{
Management of Sciatica: Conservative Versus Surgical
}

\author{
MS RAHMAN, MT UDDIN, M AHSANULLA
}

\begin{abstract}
Summary:
Many peoples suffer from sciatica, which is a mechanical pain along sciatic nerve distribution. There is lot of controversy over superiority of either conservative or surgical treatment. The aim of the present review is to compare the efficacy of both sides of treatment options. In all available studies it seems that a substantial proportion of patients improve over time. This holds true for patients undergoing surgery or receiving conservative care. Patients undergoing disc surgery are more likely to
\end{abstract}

\section{Introduction:}

Sciatica means pain along sciatic nerve distribution. The most important symptoms are radiating leg pain and related disabilities. Patients are commonly treated in primary care but a small proportion is referred to secondary care and may eventually need surgery. Many synonyms for sciatica appear in the literature, such as lumbosacral radicular syndrome, ischias, nerve root pain, and nerve root entrapment.

In about $90 \%$ of cases sciatica is caused by a herniated disc with nerve root compression, but lumbar stenoses and (less often) tumors are possible causes. ${ }^{1}$ The diagnosis of sciatica and its management varies considerably within and between countriesfor example, the surgery rates for lumbar discectomy vary widely between countries. A recent publication confirmed this large variation in disc surgery, even within countries. ${ }^{2}$ This may in part be caused by a paucity of evidence on the value of diagnostic and therapeutic interventions and a lack of clear clinical guidelines or reflect differences in healthcare and insurance systems. This review presents the current state of science for the diagnosis and treatment of sciatica

Dr. Md. Shahidur Rahman, Assistant Professor, Dr. Mohd. Taslim Uddin, Associate Professor, Dr. Md. Ahsanulla, Assistant Professor, Department of Physical Medicine and Rehabilitation, Bangabandhu Shiekh Mujib Medical University, Dhaka.

Address of Correspondence: Dr. Md. Shahidur Rahman, Assistant Professor, Department of Physical Medicine and Rehabilitation, Bangabandhu Shiekh Mujib Medical University, Email: shahidurphysmed@yahoo.com

Received: 5 June, 2008

Accepted: 7 August, 2008 get quicker relief of leg symptoms than patients receiving conservative care. If symptoms do not improve after 6-8 weeks, patients may opt for disc surgery. Those who are hesitant about surgery and can cope with their symptoms may opt for continued conservative care. Patient preference is therefore an important feature in the decision process.

Key words: Sciatica, management, conservative, surgical.

(J Bangladesh Coll Phys Surg 2008; 26: 142-146)

\section{Epidemiological perspective}

Exact data on the incidence and prevalence of sciatica are lacking. In general an estimated $5 \%-10 \%$ of patients with low back pain have sciatica, whereas the reported lifetime prevalence of low back pain ranges from $49 \%$ to $70 \%$. The annual prevalence of disc related sciatica in the general population is estimated at $2.2 \%$. ${ }^{3} \mathrm{~A}$ few personal and occupational risk factors for sciatica have been reported (box 1), including age, height, mental stress, cigarette smoking, and exposure to vibration from vehicles. ${ }^{3,4}$ Evidence for an association between sciatica and sex or physical fitness is conflicting. ${ }^{3,4}$

\section{Box 1}

Risk factors for acute sciatica ${ }^{3}$ Personal factors

- Age (peak 45-64 years)

- Increasing risk with height

- Smoking

- Mental stress

- Occupational factors

- Strenuous physical activity - for example, frequent lifting, especially while bending and twisting

- Driving, including vibration of whole body

\section{Diagnosis of sciatica}

Sciatica is mainly diagnosed by history taking and physical examination. By definition patients mention radiating pain in the leg. They may be asked to report the distribution of the pain and whether it radiates 
below the knee and drawings may be used to evaluate the distribution. Sciatica is characterised by radiating pain that follows a dermatomal pattern. Patients may also report sensory symptoms.

Physical examination largely depends on neurological testing. The most applied investigation is the straight leg raising test or Lasègue's sign. Patients with sciatica may also have low back pain but this is usually less severe than the leg pain. The diagnostic value of history and physical examination has not been well-studied ${ }^{5}$. No history items or physical examination tests have both high sensitivity and high specificity. The pooled sensitivity of the straight leg raising test is estimated to be $91 \%$, with a corresponding pooled specificity of $26 \%$. ${ }^{6}$ The only test with a high specificity is the crossed straight leg raising test, with a pooled specificity of $88 \%$ but sensitivity of only $29 \% .{ }^{6}$ Overall, if a patient reports the typical radiating pain in one leg combined with a positive result on one or more neurological tests indicating nerve root tension or neurological deficit the diagnosis of sciatica seems justified.

Value of imaging Diagnostic imaging is only useful if the results influence further management. In acute sciatica the diagnosis is based on history taking and physical examination and treatment is conservative (non-surgical). Imaging may be indicated at this stage only if there are indications or "red flags" that the sciatica may be caused by underlying disease (infections, malignancies) rather than disc herniation.

Diagnostic imaging may also be indicated in patients with severe symptoms who fail to respond to conservative care for 6-8 weeks. In these cases surgery might be considered and imaging used to identify if a herniated disc with nerve root compression is present and its location and extent. It is important as part of the decision to operate that the clinical findings and symptoms correspond well with the scan findings. This is especially relevant because disc herniations identified by computed tomography or magnetic resonance imaging are highly prevalent $(20 \%-36 \%)$ in people without symptoms who do not have sciatica 7 In many people with clinical symptoms of sciatica no lumbar disc herniations are present on scans. ${ }^{8,9}$ At present no one type of imaging method shows a clear advantage over others.
Although some authors favor magnetic resonance imaging above other imaging techniques because computed tomography has a higher radiation dose or because soft tissues are better visualized, 10, 11 evidence shows that both are equally accurate at diagnosing lumbar disc herniation. ${ }^{12}$ Radiography for the diagnosis of lumbar disc herniation is not recommended because discs cannot be visualized by X rays. ${ }^{12}$

\section{Course of acute sciatica}

In general the clinical course of acute sciatica is favorable and most pain and related disability resolves within two weeks. For example, in a randomized trial that compared non-steroidal antiinflammatory drugs with placebo for acute sciatica in primary care $60 \%$ of the patients recovered within three months and 70\% within 12 months. ${ }^{13}$ About $50 \%$ of patients with acute sciatica included in placebo groups in randomized trials of non-surgical interventions reported improvement within 10 days and about $75 \%$ reported improvement after four weeks. ${ }^{14}$ In most patients therefore the prognosis is good, but at the same time a substantial proportion (up to $30 \%$ ) continues to have pain for one year or longer. 13,14

\section{Efficacy of conservative treatments for sciatica}

Conservative treatment for sciatica is primarily aimed at pain reduction, either by analgesics or by reducing pressure on the nerve root. A recent systematic review found that conservative treatments do not clearly improve the natural course of sciatica in most patients or reduce symptoms. ${ }^{15}$ Adequately informing patients about the causes and expected prognosis may be an important part of the management strategy. However, educating patients about sciatica has not been specifically investigated in randomized controlled trials.

Box bellow summarizes the evidence of effectiveness for commonly available conservative treatments for sciatica, including injection therapy. Strong evidence of effectiveness is lacking for most of the available interventions. Little difference in effect on pain and functional status has been shown between bed rest and advice on staying active. ${ }^{16}$ As a result of this finding, bed rest-for a long time the mainstay of treatment for sciatica-is no longer widely 
recommended. Analgesics, non-steroidal antiinflammatory drugs, and muscle relaxants do not seem to be more effective than placebo in reducing symptoms. Evidence for opoids and various compound drugs is lacking. A systematic review reported that no evidence exists for traction, nonsteroidal anti-inflammatory drugs, intramuscular steroids, or tizanidine being superior to placebo. ${ }^{14}$ This review suggested that epidural injections of steroid might be effective in patients with acute sciatica. ${ }^{14}$ However a more recent systematic review of a larger number of randomized trials reported that there was no evidence of positive short-term effects of corticosteroid injections and that the long-term effects were unknown. 15 The same systematic review reported that active physical therapy (exercises) seemed not to be better than inactive (bed rest) treatment and other conservative treatments, such as traction, manipulation, hot packs, or corsets). ${ }^{15}$

\section{Box-2}

Levels of evidence for conservative treatments for sciatica $^{16}$

- Bed rest (trade-off)

- Staying active, in contrast to bed rest (likely to be beneficial)

- Analgesics or non-steroidal anti-inflammatory drugs, acupuncture, epidural steroid injections, spinal manipulation, traction therapy, physical therapy, behavioural treatment, multidisciplinary treatment (unknown effectiveness)

\section{Role of surgery in sciatica}

Surgical intervention for sciatica focuses on removal of disc herniation and eventually part of the disc or on foraminal stenosis, with the purpose of eliminating the suspected cause of the sciatica. Treatment is aimed at easing the leg pain and corresponding symptoms and not at reducing the back pain. Consensus is that a cauda equina syndrome is an absolute indication for immediate surgery. Elective surgery is the choice for unilateral sciatica. Until recently only one relatively old randomized trial was available that compared surgical intervention with conservative treatment for patients with sciatica. ${ }^{17}$ This study showed that surgical intervention had better results after one year, whereas after four and 10 years of follow-up no significant differences were found. ${ }^{17}$ A Cochrane review summarized the available randomized clinical trials evaluating disc surgery and chemonucleolysis. 18. In chemonucleolysis the enzyme chymopapain is injected in the discus with the purpose of shrinking the nucleus pulposus. The review reported better results with disc surgery than with chemonucleolysis in patients with severe sciatica of relatively long duration varying from more than four weeks to more than four months. Chemonucleolysis was more effective than placebo. Indirectly therefore the review suggested that disc surgery is more effective than placebo. On the basis of data from three trials the authors concluded that evidence is considerable that surgical discectomy provides effective clinical relief for carefully selected patients with sciatica as a result of lumbar disc prolepses that fails to resolve with conservative care. A recent review came to the same conclusion. 19. The Cochrane review further concluded that the long-term effects of surgical intervention are unclear and that evidence on the optimal timing of surgery is also lacking. ${ }^{18}$.

\section{Comparing disc surgery with conservative treatment}

Two additional randomized controlled trials have been published comparing disc surgery with conservative treatment. One trial $(n=56)$ compared microdiscectomy with conservative treatment in patients who had had sciatica for six to 12 weeks. ${ }^{20}$ Overall, no significant differences were found for leg pain, back pain, and subjective disability over two years of follow-up. Leg pain; however, seemed to initially improve more rapidly in patients in the discectomy group. The large spine patient outcomes research trial (a randomized trial) and related observational cohort study was carried out in the United States. ${ }^{21,22}$ Patients with sciatica for at least six weeks and confirmed disc herniation were invited to participate in either a randomized trial or an observational cohort study. Patients in the trial were randomized to disc surgery or to conservative care. Patients in the cohort study received disc surgery or conservative care based on their preference. In the randomized trial $(n=501)$ both treatment groups improved substantially over two years for all primary 
and secondary outcome measures. Small differences were found in favour of the surgery group, but these were not statistically significant for the primary outcome measures. Only $50 \%$ of the patients randomized to surgery received surgery within three months of inclusion compared with $30 \%$ randomized to conservative care. After two years of follow-up $45 \%$ of patients in the conservative care group underwent surgery compared with $60 \%$ in the surgery group. ${ }^{21}$ The observational cohort included 743 patients. Both groups improved substantially over time, but the surgery group showed significantly better results for pain and function compared with the conservative group. The authors did mention caution in interpreting the findings because of potential confounding by indication and because outcome measures were self reported. ${ }^{22}$ The results indicate that both conservative care and disc surgery are relevant treatment options for patients with sciatica of at least six weeks' duration. Surgical intervention may provide quicker relief of symptoms compared with conservative care, but no large differences have been found in success rate after one or two years of follow-up. Patients and doctors may thus weigh the benefits and harms of both options to make individual choices. This is especially relevant because patients' preference for treatment may have a direct positive influence on the magnitude of the treatment effect.

\section{Recommendations in clinical guidelines}

Although in many countries clinical guidelines are available for the management of non-specific low back pain this is not the case for sciatica. ${ }^{23}$ After excluding specific diseases on the basis of red flags, sciatica is diagnosed on the basis of history taking and physical examination. Initial treatment is conservative, with a strong focus on patient education, advice to stay active, continuing daily activities, and adequate treatment for pain. In this phase imaging has no role. Referral to a medical specialist-for example, neurologist, rheumatologist, and spine surgeon - is indicated in patients whose symptoms do not improve after conservative treatment for at least 6-8 weeks. In these referred cases surgery may be considered. Immediate referral is indicated in cases with a cauda equina syndrome. Acute severe paresis or progressive paresis is also reasons for referral within a few days.

\section{Future research}

More information is needed on the importance of clinical signs and symptoms for the prognosis of sciatica and the response to treatment. This includes the value of size and location of the disc herniation, visible nerve root compression, sequestration, and the results of history taking and physical and neurological examinations. Subgroup analysis in a Finnish trial showed that discectomy was superior to conservative treatment in patients with disc herniation at L4-5. ${ }^{24}$ No strong evidence exists for or against the efficacy of many of the available conservative treatments. Much progress can be achieved here. Questions remain about the efficacy of analgesics for sciatica and the value of physical therapy and of patient education and counseling. No trial has yet evaluated the effectiveness of behavioral treatment and multidisciplinary treatment programmes.

Tumor necrosis factor has been identified in animal and human studies as one factor in the development of sciatica. ${ }^{24,25}$ The first randomized trial evaluating a tumor necrosis factor antagonist in patients with sciatica did not find a positive result. ${ }^{26}$

\section{Conclusion:}

More evidence-based information has become available on the efficacy of surgical care compared with conservative care for patients with sciatica. Although evidence is limited, initial findings suggest no important differences in long term (one or two years) effect between these two approaches. This finding may be partly explained by patients who initially received conservative care later undergoing disc surgery. In all available studies it seems that a substantial proportion of patients improve over time. This holds true for patients undergoing surgery or receiving conservative care. Patients undergoing disc surgery are more likely to get quicker relief of leg symptoms than patients receiving conservative care. If symptoms do not improve after 6-8 weeks patients may opt for disc surgery. Those who are hesitant about surgery and can cope with their symptoms may opt for continued conservative care. Patient preference is therefore an important feature in the decision process.

Since the mid-1990s a switch has occurred in the management of sciatica from passive treatments, such as bed rest, to a more active approach, with patients being advised to continue their daily activities as 
much as possible. Our experience advocates the use of conservative treatment for at least two to three weeks except the patients that suffer from cauda equina syndrome or can not cope with the pain and want surgery despite adequate analgesics and physiatric measures. Mere neurological deficit should not be the indication for surgery as we have seen most neurological deficits improve by six weeks.

\section{References:}

1. B W Koes, M W van Tulder. Diagnosis and treatment of sciatica BMJ 2007; 334: 1313-1317

2. Weinstein JN, Lurie JD, Olson PR, Bronner KK, Fisher ES United States' trends and regional variations in lumbar spine surgery: 1992-2003. Spine 2006; 31: 2707-14.

3. Younes M, Bejia I, Aguir Z, Letaief M, Hassen-Zroer S, Touzi M, et al. Prevalence and risk factors of disc-related sciatica in an urban population in Tunisia. Joint Bone Spine 2006; 73: 538-42

4. Miranda H, Viikari-Juntera E, Martikainen R, Takala E, Riihimaki H. Individual factors, occupational loading, and physical exercise as predictors of sciatic pain. Spine 2002; 27: $1102-9$

5. Vroomen PCAJ, Krom MCTFM de, Knottnerus JA Diagnostic value of history and physical examination in patients suspected of sciatica due to disc herniation: a systematic review. J Neurol 1999; 246: 899-906

6. Deville WLJM, Windt DAWM, van der Dzaferagic A, Bezemer PD, Bouter LM. The test of Lasegue: systematic review of the accuracy in diagnosing herniated discs. Spine 2000; 25: 1140-7

7. Jensen MC, Brant-Zawadzki MN, Obuchowski N, Modic MT, Malkasian D, Ross JS. Magnetic resonance imaging of the lumbar spine in people without back pain. N Engl J Med 1994; 331: 69-73

8. Modic MT, Ross JS, Obuchowski NA, Browning KH, Cianflocco AJ, Mazanec DJ. Contrast-enhanced MR imaging in acute lumbar radiculopathy: a pilot study of the natural history. Radiology 1995; 195: 429-35

9. Modic MT, Obuchowski NA, Ross J, Brant-Zawadzki MN, Grooff PN, Mazanec DJ, et al. Acute low back pain and radiculopathy: MR imaging findings and their prognostic role and effect on outcome. Radiology 2005; 237: 597-604

10. Govind J. Lumbar radicular pains. Aus Fam Phys 2004; 33 409-12.

11. Awad JN, Moskovich R. Lumbar disc herniations: surgical versus nonsurgical treatment. Clin Orthop Relat Res 2006; 443: $183-97$

12. Jarvik JG, Deyo RA. Diagnostic evaluation of low back pain with emphasis on imaging. Ann Intern Med 2002; 137 586-97.
13. Weber H, Holme I, Amlie E. The natural course of acute sciatica with nerve root symptoms in a double blind placebo-controlled trial of evaluating the effect of piroxicam (NSAID). Spine 1993; 18: 1433-8

14. Vroomen PCAJ, Krom MCTFM de, Slofstra PD, Knottnerus JA. Conservative treatment of sciatica: a systematic review. J Spinal Dis 2000; 13: 463-9

15. Luijsterburg PAJ, Verhagen AP, Ostelo RWJG, Os TAG van, Peul WC, Koes BW. Effectiveness of conservative treatments for the lumbosacral radicular syndrome: a systematic review. Eur Spine J 2007 Apr 6; (Epub ahead of print)

16. Hagen KB, Jamtvedt G, Hilde G, Winnem MF. The updated Cochrane review of bedrest for low back pain and sciatica. Spine 2005; 30: 542-6

17. Weber H. Lumbar disc herniation. A controlled prospective study with ten years of observation. Spine 1983; 8: 131-40

18. Gibson JN, Waddell G. Surgical interventions for lumbar disc prolapse. Cochrane Database Syst Rev 2007 Jan 24; (1): CD001350.

19. Van Tulder MW, Koes B, Seitsalo S, Malmivaara A. Outcome of invasive treatment modalities on back pain and sciatica: an evidence-based review. Eur Spine J 2006; 15: S82-92

20. Osterman H, Seitsalo S, Karppinen J, Malmivaara A. Effectiveness of microdiscectomy for lumbar disc herniation. Spine 2006; 31: 2409-14

21. Weinstein JN, Tosteson TD, Lurie JD, Tosteson ANA, Hanscom B, Skinner JS, et al. Surgical vs nonoperative treatment for lumbar disk herniation: the spine patient outcomes research trial (SPORT): a randomized trial. JAMA 2006; 296: 2441-50

22. Weinstein JN, Lurie JD, Tosteson TD, Skinner JS, Hanscom B, Tosteson ANA, et al. Surgical vs nonoperative treatment for lumbar disk herniation: the spine patient outcomes research trial (SPORT) observational cohort. JAMA 2006; 296: 2451-9

23. Koes BW, Tulder MW van, Ostelo R, Burton AK, Waddell G. Clinical guidelines for the management of low back pain in primary care: an international comparison. Spine 2001; 26: $2504-13$

24. Mulleman D, Mammou S, Griffoul I, Watier H, Goupille P. Pathophysiology of disc-related sciatica. I. Evidence supporting a chemical component. Joint Bone Spine 2006; 73: $151-8$

25. Mulleman D, Mammou S, Griffoul I, Watier H, Goupille P. Pathophysiology of disc-related low back pain and sciatica. II. Evidence supporting treatment with TNF-alfa antagonists. Joint Bone Spine 2006; 73: 270-7

26. Korhonen T, Karppinen J, Paimela L, Malmivaara A Lindgren KA, Bowman $\mathrm{C}$, et al. The treatment of disc herniation-induced sciatica with infliximab: one-year follow-up results of FIRST II, a randomized controlled trial. Spine 2006; 31: 2759-66 